mortality and present a significant diagnostic challenge to the clinical microbiologist. This case-based presentation will present a spectrum of these diverse esoteric parasitic infections to challenge and stimulate the audience. Come prepared to think outside of the box and see if you can make the correct diagnosis!

References

1. United States Centers for Disease Control and Prevention. Parasites. Cited 16 Oct 2019. https://www.cdc.gov/parasites/index.html.

2. Cox FE. History of human parasitology. Clin Microbiol Rev 2002; 15 : 595-612.

3. World Health Organization. Neglected parasitic diseases. Cited 16 Oct 2019. https://www.who.int/neglected_diseases/en/.

\section{REFRACTORY FACIAL CUTANEOUS LEISHMANIASIS IN A CHILD}

David Foley, Tim Ford, Anita Campbell, Aleisha Anderson, Briony Hazelton, Daniel Yeoh

Infectious Diseases, Perth Children's Hospital, WA, Australia

Cutaneous leishmanasis (CL) is the most common form of leishmaniasis. The lesions are typically painless and can selfresolve. Chronic cases occur, particularly in immunocompromised patients. This report describes the treatment of a paediatric patient with refractory cutaneous leishmaniasis. Treatment of CL in children requires consideration of drug dosing, palatability, ethics of utilising treatments with limited supporting evidence and the potential long-term side effects.

An immunocompetent 13-year-old girl presented with nonhealing ulcer on her face following migration from Pakistan in 2018. The lesion had persisted despite previous empiric treatment with intra-lesional sodium stibogluconate. Biopsy was performed and Leishmaniasis tropica identified by PCR. Response to topical paromomycin was poor. There was partial resolution with oral miltefosine $50 \mathrm{mg}$ BD for 28 days. Re-ulceration occurred within 6 weeks of cessation. The use of liposomal amphotericin was precluded by initial anaphylaxis; desensitisation was unsuccessful. Miltefosine was restarted at a higher dose of $50 \mathrm{mg}$ TDS combined with fluconazole $300 \mathrm{mg}$ daily. Again there was partial response but diminished compared to previous. After 3 months, fluconazole was replaced with allopurinol $200 \mathrm{mg}$ TDS for 30 days. The lesion remained static. Topical GM-CSF was subsequently added for 3 weeks, with marginal improvement. Finally, photodynamic therapy was initiated in December 2019.

\section{A DIAGNOSTIC FLUKE}

Jane Dyer $^{1,2}$, Andrew Henderson ${ }^{2,3}$

${ }^{T}$ Department of Microbiology, Pathology Queensland, Brisbane, Australia; ${ }^{2}$ School of Medicine, University of Queensland, Brisbane, Australia; and ${ }^{3}$ Infection Management Services, Metro South Health, Brisbane, Australia

Fascioliasis is a foodborne trematode infection caused by the liver fluke Fasciola hepatica or Fasciola gigantica. In Australia, human cases are seen rarely and infection is often acquired overseas. This study reports the case of an 18-year-old woman from Bangladesh, with a mulitloculated liver abscess suspected secondary to $F$. hepatica. She presented with fever and right upper quadrant pain, and initial blood examination at our facility revealed an eosinophilia of $1.22 \times 10^{9} / \mathrm{L}(9 \%)$ and a mild elevation of liver function tests. An ultrasound guided drainage was sent only for cytology which revealed no malignancy. Empiric cover was given for bacterial liver abscess and blood cultures were negative. Multiple stool microscopic examinations visualised no ova, cysts or parasites. Serology demonstrated reactivity for Fasciola hepatica IgG enzyme immunoassay (EIA) and Schistosomiasis IgG EIA which was treated with praziquantel. There was non-reactive serology for Entamoeba histolytica, Echinococcus granulosus and Strongyloides. Despite treatment with triclabendazole, her case was later complicated by acute bleeding through the ampulla of vater secondary to a hepatic artery pseudoaneurysm and she received re-treatment with triclabendazole. Although we cannot differentiate between other liver flukes as the cause of her presentation, this case highlights important microbiological diagnostic considerations and treatment challenges.

\section{POLIO WILL GO, AFM WILL STAY - A CASE REPORT}

\section{Syeda Naqvi}

Department of Microbiology, John Hunter Laboratory, NSW

Health Pathology, NSW, Australia

Background: Poliovirus related acute flaccid myelitis (AFM) was one of the most feared diseases. Due to effective vaccines, poliovirus is at the verge of global eradication. However, recent world-wide outbreaks of AFM cases associated with Enterovirus-D68 (EV-D68) are stimulating concerns about a new 'polio-like' epidemic, challenging mankind with the devastating outcomes, primarily in children.

Case: A previously healthy, 5-year-old boy presented in paediatric ICU with sudden onset upper limbs weakness and breathing difficulty. Two days earlier, he had an upper respiratory illness with mild fever. On examination, he had hypotonia of truncal and limb muscles, with predominant loss of proximal power in the upper limbs. Intubation and mechanical ventilation were required for respiratory failure. MRI spine revealed myelitic changes. Respiratory viral PCR on nasopharyngeal swab was positive for Enterovirus. CSF had mildly raised WCC and proteins but was negative for viruses. EV-D68 was identified from faecal specimens by VP1 region sequencing at the National Enterovirus Reference Laboratory. Despite immunomodulatory therapy and intensive inpatient physiotherapy, the patient had ventilator dependence and a significant residual weakness in his upper limbs.

Conclusion: EV-D68 is a neurotropic virus. Increasing awareness amongst clinicians, proper diagnostics and integrating EVD68 into accessible surveillance systems will be essential to reveal the burden of disease.

\section{References}

1. Cassidy H, Poelman R, Knoester M. Enterovirus D68 - the new polio? Front Microbiol 2018; 9: 2677.

2. Uprety P, Curtis D, Elkan M, et al. Association of Enterovirus D68 with acute flaccid myelitis. Emerging Infectious Diseases 2019; 25: $1676-82$.

\section{A CASE OF LEGIONELLA ENDOCARDITIS ASSOCIATED WITH CARDIAC SURGERY}

Aaron Keene

Department of Microbiology, Canterbury Health Labs, Christchurch Hospital, New Zealand 